\title{
DOA Estimation for Multi-Band Signal Sources Using Compressed Sensing Techniques with Khatri-Rao Processing*
}

\author{
Tsubasa TERADA ${ }^{\dagger}$, Student Member, Toshihiko NISHIMURA ${ }^{\dagger a}{ }^{\text {a }}$, Member, Yasutaka OGAWA $^{\dagger}$, Fellow , \\ Takeo OHGANE ${ }^{\dagger}$, Member, and Hiroyoshi YAMADA ${ }^{\dagger \dagger}$, Senior Member
}

\begin{abstract}
SUMMARY Much attention has recently been paid to direction of arrival (DOA) estimation using compressed sensing (CS) techniques, which are sparse signal reconstruction methods. In our previous study, we developed a method for estimating the DOAs of multi-band signals that uses CS processing and that is based on the assumption that incident signals have the same complex amplitudes in all the bands. That method has a higher probability of correct estimation than a single-band DOA estimation method using CS. In this paper, we propose novel DOA estimation methods for multi-band signals with frequency characteristics using the Khatri-Rao product. First, we formulate a method that can estimate DOAs of multiband signals whose phases alone have frequency dependence. Second, we extend the scheme in such a way that we can estimate DOAs of multi-band signals whose amplitudes and phases both depend on frequency. Finally, we evaluate the performance of the proposed methods through computer simulations and reveal the improvement in estimation performance.

key words: DOA estimation, multi-band signal, compressed sensing, Khatri-Rao product, sparse signal reconstruction
\end{abstract}

\section{Introduction}

There have been various kinds of studies on direction of arrival (DOA) estimation [2]. These techniques have been applied to a wide range of fields, such as radar systems, positioning systems, and communication quality improvement. For these techniques to advance, the estimation accuracy must be improved. The beamformer and the Capon [3] algorithms are fundamental estimation techniques. The MUSIC algorithm [4] and ESPRIT [5] have been proposed as high resolution estimation techniques. The EM [6], [7] and SAGE [8] algorithms based on a maximum likelihood method also have been used for DOA estimation as a super resolution technique, although the computational load is larger than for MUSIC and ESPRIT. Recently, much attention has been paid to DOA estimation using compressed sensing (CS) techniques [9]-[11], which have been used mainly in the areas of signal processing, image compression, and wireless communication networks. A technique to estimate DOAs of ultrawideband (UWB) signals is also effective at improving the estimation accuracy [12].

\section{Manuscript received February 3, 2014}

Manuscript revised May 14, 2014.

${ }^{\dagger}$ The authors are with the Graduate School of Information Science and Technology, Hokkaido University, Sapporo-shi, 0600814 Japan.

${ }^{\dagger}$ The author is with the Graduate School of Science \& Technology, Niigata University, Niigata-shi, 950-2181 Japan.

*The basic concept of this scheme was presented in [1]. Detailed results are presented in this paper.

a)E-mail: nishim@ist.hokudai.ac.jp

DOI: 10.1587/transcom.E97.B.2110
$\mathrm{CS}$ is a signal processing technique that allows the reconstruction of sparse signals from a few measurements [13]. A general DOA estimation method using CS techniques is described below. First, we divide the space surrounding receiving antennas in the angle domain, and we call the divided partitions 'bins'. Second, we set an original signal as a complex amplitude of an incident wave from each bin. If a wave arrives from a bin, the complex amplitude corresponding to the bin has a non-zero value. In contrast, complex amplitudes in the other bins that do not have incident waves are zero. Finally, we reconstruct the original signals by applying a CS technique to the data obtained from the receiving antennas. Each original signal has the complex amplitude in each bin, and we can obtain DOAs from the reconstructed original signals.

We have proposed DOA estimation of multi-band signals using a CS technique [14]. In the system, each source transmits signals over multiple frequency bands, and the receiver side estimates the directions of the sources. The results show an improvement in the probability of correct estimation. However, we assumed that incident waves have multiple bands whose complex amplitudes are the same. In this paper, using the Khatri-Rao matrix product [15], [16] we propose methods that enable DOA estimation of multiband signals with different complex amplitudes in different bands. Because the Khatri-Rao product does not consist of complex amplitudes but of powers, the frequency characteristics of phases do not affect the estimation accuracy. We also propose power adjustment in such a way that we can avoid the frequency characteristics of power and amplitude. Thus, we can estimate DOAs of multi-band signals with frequency dependent complex amplitudes.

In a UWB system, if we have narrow-band filters or a filter bank as will be shown later in this paper, we can regard the system as a multi-band transmission one, and the proposed technique can be applied. In future, a multi-band signal system might be introduced for precise positioning. The scheme proposed in this paper may play an important role in that case.

In this paper, we assume that direct waves alone are dominant, and that we can neglect refracted waves, diffracted ones, and reflected ones. Thus, all the multi-band components emitted from a signal source arrive from the same direction.

This paper is organized as follows. In Sect. 2, we show common DOA estimation using a CS technique that recon- 


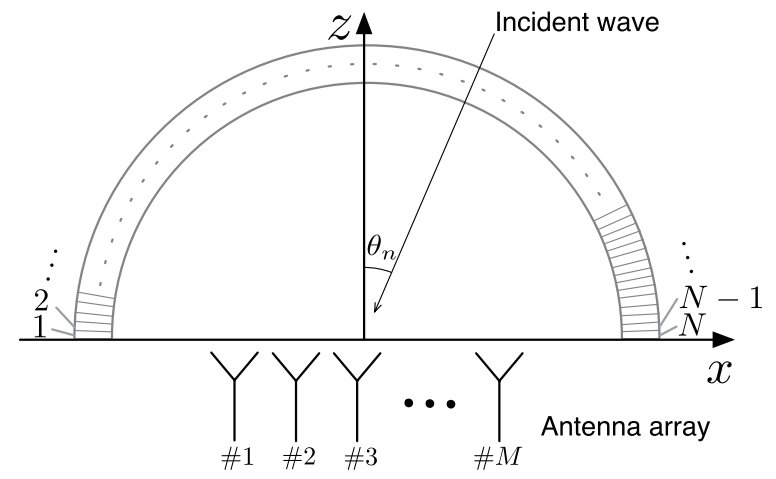

Fig. 1 DOA model.

structs complex amplitudes of signals. We propose methods that reconstruct the powers of the signals in Sect. 3. In Sect. 4, computer simulation results are presented. We give some concluding remarks in Sect. 5 .

\section{DOA Estimation Using Compressed Sensing Tech- nique}

\subsection{DOA Model}

A DOA model of a wave impinging upon a receiving antenna array is shown in Fig. 1. For easy understanding, we first consider narrowband source signals in the far field impinging upon a linear array with $M$ omnidirectional antenna elements. Here, $N$ denotes the number of space partitions (bins) in the angle domain.

We express an $N$-dimensional signal vector as $\mathbf{x}(s)$, whose elements indicate the complex amplitude of an incident wave from each direction. The value of an element is zero if there is no incident wave in the corresponding bin.

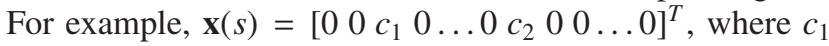
and $c_{2}$ are complex amplitudes at the corresponding bins, and $[\cdot]^{T}$ denotes the transpose. The $M$-dimensional received signal vector $\mathbf{y}(s)$ can be expressed as

$$
\mathbf{y}(s)=\mathbf{A x}(s)+\mathbf{n}(s),
$$

where $\mathbf{n}(s)$ denotes the $M$-dimensional measurement noise vector whose elements are additive white Gaussian noise having power of $\sigma^{2}$ and $s$ is sampling time. $\mathbf{A}$ is an $M \times N$ mode matrix, and the $m$ th row and $n$th column element of $\mathbf{A}$ is given by

$$
a_{f_{l}, m, \theta_{n}}=e^{-j \frac{2 \pi}{\lambda_{l}} d_{m} \sin \theta_{n}}
$$

where $\lambda_{l}$ is the wavelength for the frequency $f_{l}$. We deal with multiple frequencies $\left(f_{1}, f_{2}, \ldots\right)$ in this paper, and we introduced the subscript $l$ for the wavelength and frquency. Also, $d_{m}$ is the distance between the $m$ th antenna and the reference point, and $\theta_{n}$ denotes the direction angle corresponding to the $n$th partitioned bin.

\subsection{Compressed Sensing Technique}

CS techniques are a framework for reconstructing high- dimensional signals from a few measurements. Suppose that a vector $\mathbf{y} \in \mathbb{C}^{M}$ is a linear transformation of an unknown vector $\mathbf{x} \in \mathbb{C}^{N}$; the vector $\mathbf{y}$ can be expressed as

$$
\mathbf{y}=\mathbf{A} \mathbf{x},
$$

where $\mathbf{A} \in \mathbb{C}^{M \times N}$. This equation corresponds to (1) neglecting the noise vector $\mathbf{n}(s)$.

CS techniques deal with the reconstruction of the original signal vector $\mathbf{x}$ using the known vector $\mathbf{y}$ and the matrix A. The necessary and sufficient condition to reconstruct the vector $\mathbf{x}$ is $\operatorname{rank}(\mathbf{A})=N$. If $M<N$, we can attain a leastnorm $\left(l_{2}\right.$-norm) solution using the Moore-Penrose pseudo inverse, but the original vector $\mathbf{x}$ may not be reconstructed.

When the vector $\mathbf{x}$ is sparse, we can reconstruct the sparsest solution by using the optimization problem:

$$
\hat{\mathbf{x}}=\underset{\mathbf{x}}{\arg \min }\|\mathbf{y}-\mathbf{A x}\|_{2}^{2}+\mu\|\mathbf{x}\|_{p}^{p},
$$

where $\hat{\mathbf{x}}$ is the estimated original signal vector, $\mu$ is a nonnegative parameter, and $\|\cdot\|_{p}$ denotes the $l_{p}$-norm defined as

$$
\|\mathbf{z}\|_{p} \triangleq\left(\sum_{k=1}^{K}\left|z_{k}\right|^{p}\right)^{1 / p} .
$$

In spectrum analysis, it has been shown that the $p<2$ case results in higher resolution spectral estimates than the $p=2$ case [17].

In DOA estimation cases, we can assume that $\mathbf{x}$ is sparse. This is because the number of arriving waves is clearly much smaller than the number of partitions $N$ shown in Fig. 1. In order to reconstruct the original signal $\mathbf{x}(s)$, we formulate the optimization problem from (1) and (4):

$$
\underset{\hat{\mathbf{x}}}{\arg \min }\left\{\frac{1}{S}\left(\sum_{s=1}^{S}\|\mathbf{y}(s)-\mathbf{A} \hat{\mathbf{x}}\|_{2}^{2}\right)+\mu\|\hat{\mathbf{x}}\|_{p}^{p}\right\},
$$

where $S$ is the number of snapshots.

\subsection{Half-Quadratic Regularization Method}

In this paper, we use the half-quadratic regularization method [11] to solve (6):

$$
\mathbf{H}\left(\hat{\mathbf{x}}^{(i)}\right) \hat{\mathbf{x}}^{(i+1)}=\frac{1}{S}\left(\sum_{s=1}^{S} \mathbf{A}^{H} \mathbf{y}(s)\right),
$$

where $i$ denotes the iteration number, $\hat{\mathbf{x}}^{(i)}$ denotes the estimated original signal vector at the $i$ th iteration, and $(\cdot)^{H}$ denotes the Hermitian matrix transpose. Here, we define the following matrix:

$$
\mathbf{H}(\mathbf{z}) \triangleq \mathbf{A}^{H} \mathbf{A}+\mu \mathbf{\Lambda}(\mathbf{z}),
$$

where $\boldsymbol{\Lambda}(\mathbf{z})$ is defined as

$$
\boldsymbol{\Lambda}(\mathbf{z}) \triangleq \operatorname{diag}\left\{\frac{p / 2}{\left(\left|z_{k}\right|^{2}+\epsilon\right)^{1-p / 2}}\right\},
$$




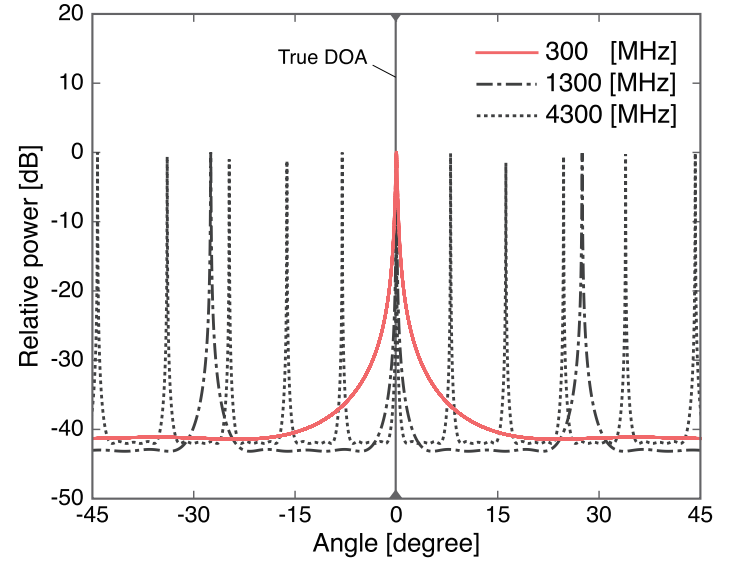

Fig. 2 MUSIC spectra at three different frequencies.

where $\operatorname{diag}\{\cdot\}$ is a diagonal matrix, and $\epsilon$ is a parameter used to avoid the problem of non-differentiability in the $l_{p}$-norm.

\subsection{Application to Multi-Band Signals}

Let us consider a difficult problem in a case of DOA estimation of a single-band signal before dealing with a multiband signal. MUSIC spectra at three different frequencies are shown in Fig. 2. The true angle of the incident signal is $0^{\circ}$ and the frequencies are $300 \mathrm{MHz}, 1300 \mathrm{MHz}$, and $4300 \mathrm{MHz}$. We assumed a uniform linear array (ULA) with five antenna elements at the receiver. The antenna spacing is $0.5 \mathrm{~m}$, which corresponds to half the wavelength for $300 \mathrm{MHz}$. The SNR is fixed at $20 \mathrm{~dB}$ and the number of snapshots for each estimation is 100 . From the figure, we can see that the higher the frequency is, the more peaks other than the true DOA appear. It can be clearly seen that these unnecessary peaks due to grating lobes negatively affect the correct DOA estimation. The grating lobes are generated when antenna spacing is larger than half the wavelength of the frequency ${ }^{\dagger}$. The positions of the grating lobes for the true DOA $\theta$ are given by

$$
\theta_{G L}=\sin ^{-1}\left(\sin \theta+\frac{\lambda_{l}}{d} q\right) \quad(q= \pm 1, \pm 2, \cdots) .
$$

where $d$ is the antenna spacing. This problem is solved by the CS technique that will be stated later.

In this section, we assume that incident waves have multiple bands and that the complex amplitudes of the bands are the same for convenience.

Assuming that each incident wave has $L$ bands, the received signal vector in (1) can be expressed as

${ }^{\dagger}$ Strictly speaking, when antenna spacing is half the wavelength, we have a grating lobe. When DOA is $90^{\circ}$, we have the effect of a grating lobe at $-90^{\circ}$. They are the directions along the array axis. This is, however, a rare case. In this paper, we consider that a ULA with antenna spacing of half the wavelength does not suffer from grating lobes.

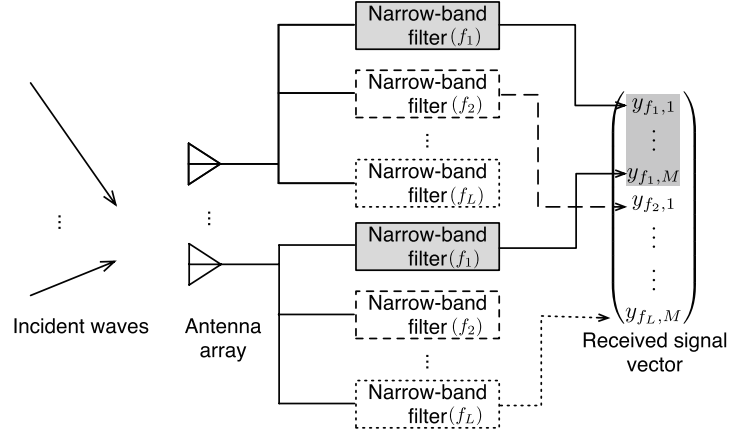

Fig. 3 Concept of extracting narrow-band signal components from multi-band signals.

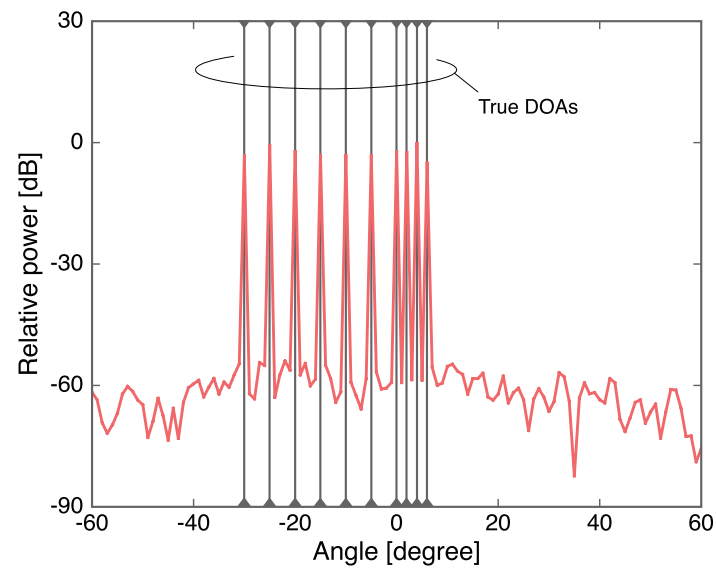

Fig. 4 DOA estimation by using the CS technique for multi-band signals.

$$
\left(\begin{array}{c}
\mathbf{y}_{f_{1}}(s) \\
\mathbf{y}_{f_{2}}(s) \\
\vdots \\
\mathbf{y}_{f_{L}}(s)
\end{array}\right)=\left(\begin{array}{c}
\mathbf{A}_{f_{1}} \\
\mathbf{A}_{f_{2}} \\
\vdots \\
\mathbf{A}_{f_{L}}
\end{array}\right) \mathbf{x}(s)+\left(\begin{array}{c}
\mathbf{n}_{f_{1}}(s) \\
\mathbf{n}_{f_{2}}(s) \\
\vdots \\
\mathbf{n}_{f_{L}}(s)
\end{array}\right),
$$

where $\mathbf{y}_{f_{l}}(s)$ is the received signal vector for the $l$ th band. Also, $\mathbf{A}_{f_{l}}$ and $\mathbf{n}_{f_{l}}(s)$ are the mode matrix and noise vector in the $l$ th frequency band, respectively. The concept of extracting narrow band signal components from multi-band signals is shown in Fig. 3. The received signals at each antenna pass through narrow-band filters for each frequency and are divided into $L$ narrow-band signal components.

Here, we assume that the lowest band $f_{1}$ meets the requirement that grating lobes are not generated, i.e., $d \leq \lambda_{1} / 2$ holds. In this case, DOA estimation of multi-band signals using the CS technique on the basis of (11) is not susceptible to grating lobes.

The estimated relative power of multi-band signals as a function of angle for the case of ten uncorrelated incident waves is shown in Fig. 4. We assumed that each incident wave has five bands and that the center frequencies are $f_{1}=300 \mathrm{MHz}, f_{2}=1300 \mathrm{MHz}, f_{3}=2300 \mathrm{MHz}$, $f_{4}=3300 \mathrm{MHz}$, and $f_{5}=4300 \mathrm{MHz}$. The other simulation parameters are the same as those in the case of Fig. 2. We uniformly divided the space surrounding the receiving antennas into 121 partitions in the angle domain in the posi- 
tive region of the $\mathrm{z}$-axis in Fig. 1; that is, the angle step is $1^{\circ}$. The angle step can be smaller than $1^{\circ}$, and we can acquire better angle resolution. However, if the angle step is extremely small, a huge computational load is needed because the dimension of the original signal vector $\mathbf{x}$ becomes very large. In real propagation environments, we will encounter the limit of the angle step because of the effect of noise and angular spread of signal sources. Detailed consideration of the angle step is one of our future works. The number of snapshots in each frequency band is 20 ; therefore, the total number is 100 . The incident angles of the waves are $-30^{\circ}$, $-25^{\circ},-20^{\circ},-15^{\circ},-10^{\circ},-5^{\circ}, 0^{\circ}, 2^{\circ}, 4^{\circ}$, and $6^{\circ}$; the solid black lines indicate the true incident angles as a reference in Fig. 4. The SNR was fixed at $30 \mathrm{~dB}$. It is seen that this method can estimate DOAs correctly, even when the number of waves exceeds the number of array elements.

\section{Applications to Multi-Band Signals with Frequency Characteristics}

\subsection{Case Where Phases Alone Depend on Frequency}

In Sect. 2, we assumed that incident waves have multiple bands whose complex amplitudes are the same. To deal with frequency dependent multi-band signals, we introduce Khatri-Rao processing. In this subsection, we propose a DOA estimation method that can be used for the case where phases alone depend on frequency. It should be noted that amplitudes and powers are independent of frequency. We assume that the incident signals are uncorrelated in any bands. Then, the correlation matrix of the incident signals $\left(\mathbf{y}_{f_{1}}(s)^{T}, \mathbf{y}_{f_{2}}(s)^{T}, \cdots, \mathbf{y}_{f_{L}}(s)^{T}\right)^{T}$ is expressed as

$$
\overline{\mathbf{R}}=\left(\begin{array}{cccc}
\mathbf{R}_{f_{1}, f_{1}} & & & 0 \\
& \mathbf{R}_{f_{2}, f_{2}} & & \\
0 & & \ddots & \\
0 & & & \mathbf{R}_{f_{L}, f_{L}}
\end{array}\right),
$$

where $\mathbf{R}_{f_{l}, f_{l}}$ is the correlation matrix in the frequency band of $f_{l}$ and is defined as

$$
\mathbf{R}_{f_{l}, f_{l}}=E\left[\mathbf{y}_{f_{l}}(s) \mathbf{y}_{f_{l}}^{H}(s)\right] \text {. }
$$

As stated above, we assume that powers of the signals in all the bands are the same; then the powers in the bands for the signal impinging from $\theta_{n}$ can be expressed as

$$
P_{f_{1}, \theta_{n}}=P_{f_{2}, \theta_{n}}=\cdots=P_{f_{L}, \theta_{n}} \triangleq P_{\theta_{n}} \text { for any } n,
$$

where $P_{f_{l}, \theta_{n}}=E\left[x_{f_{l}, \theta_{n}}(s) x_{f_{i}, \theta_{n}}^{*}(s)\right]$ and $(\cdot)^{*}$ denotes the complex conjugate. Here, we execute vector conversion for (12) and express it as:

$$
\begin{aligned}
& \operatorname{vec}(\overline{\mathbf{R}}) \\
& =\left(\mathbf{R}_{f_{1} f_{1}}^{(1)}, \mathbf{0}, \cdots, \mathbf{0}, \mathbf{R}_{f_{1} f_{1}}^{(2)}{ }^{T}, \mathbf{0}, \cdots, \mathbf{0}, \mathbf{R}_{f_{1} f_{1}}^{(M)^{T}}, \mathbf{0}, \cdots, \mathbf{0},\right. \\
& \quad \mathbf{0}, \mathbf{R}_{f_{2} f_{2}}^{(1)}{ }^{T}, \mathbf{0}, \cdots, \mathbf{0}, \mathbf{R}_{f_{2} f_{2}}^{(2)}, \mathbf{0}, \cdots, \mathbf{0}, \mathbf{R}_{f_{2} f_{2}}^{(M)^{T}}, \mathbf{0}, \cdots, \mathbf{0}, \\
& \quad \cdots,
\end{aligned}
$$

$$
\left.\mathbf{0}, \cdots, \mathbf{0}, \mathbf{R}_{f_{L} f_{L}}^{(1)}{ }^{T}, \mathbf{0}, \cdots, \mathbf{0}, \mathbf{R}_{f_{L} f_{L}}^{(2)}{ }^{T}, \mathbf{0}, \cdots, \mathbf{0}, \mathbf{R}_{f_{L} f_{L}}^{(M)^{T}}\right)^{T},
$$

where $\mathbf{R}_{f, f_{i}}^{(m)}$ denotes the $m$ th column of $\mathbf{R}_{f_{l}, f_{l}}$. Moreover, we delete trivial zero elements corresponding to the non-block diagonal matrix elements in (12), and we get

$\mathbf{r}_{e}=\left(\mathbf{R}_{f_{1}, f_{1}}^{(1)}{ }^{T}, \cdots, \mathbf{R}_{f_{1}, f_{1}}^{(M)^{T}}, \mathbf{R}_{f_{2}, f_{2}}^{(1)}{ }^{T}, \cdots, \cdots, \mathbf{R}_{f_{L}, f_{L}}^{(M)^{T}}\right)^{T}$.

Note that $\mathbf{r}_{e}$ corresponds to the left-hand side of (1).

Now, we divide $\operatorname{vec}(\overline{\mathbf{R}})$ into a signal part and a noise part:

$$
\begin{aligned}
\operatorname{vec}(\overline{\mathbf{R}}) & =\operatorname{vec}\left(\mathbf{B S B}^{H}\right)+\operatorname{vec}\left(\overline{\mathbf{R}}_{N}\right) \\
& =\left(\mathbf{B}^{*} \odot \mathbf{B}\right) \mathbf{p}_{e}+\operatorname{vec}\left(\overline{\mathbf{R}}_{N}\right),
\end{aligned}
$$

where $\mathbf{B}$ is the $M L \times N$ mode matrix arranged vertically from $\mathbf{A}_{f_{1}}$ to $\mathbf{A}_{f_{L}}$, as given by

$$
\mathbf{B}=\left(\mathbf{A}_{f_{1}}{ }^{T}, \mathbf{A}_{f_{2}}{ }^{T}, \cdots, \mathbf{A}_{f_{L}}{ }^{T}\right)^{T} .
$$

In these expressions, $\mathbf{S}$ is the correlation matrix of $\mathbf{x}, \overline{\mathbf{R}}_{N}$ is the correlation matrix of the noise vector, and $\mathbf{p}_{e}=$ $\left(P_{\theta_{1}}, P_{\theta_{2}}, \cdots, P_{\theta_{N}}\right)^{T}$. Furthermore, $\odot$ is the operator of the Khatri-Rao product. Here, we delete trivial zero elements in the right-hand side of (17); then $\mathbf{r}_{e}$ is given by

$$
\begin{aligned}
\mathbf{r}_{e} & =\left(\begin{array}{ccc}
\overline{\mathbf{A}}_{f_{1}, \theta_{1}}^{(1)} & \cdots & \overline{\mathbf{A}}_{f_{1}, \theta_{N}}^{(1)} \\
& \vdots & \\
\overline{\mathbf{A}}_{f_{1}, \theta_{1}}^{(M)} & \cdots & \overline{\mathbf{A}}_{f_{1}, \theta_{N}}^{(M)} \\
\overline{\mathbf{A}}_{f_{2}, \theta_{1}}^{(1)} & \cdots & \overline{\mathbf{A}}_{f_{2}, \theta_{N}}^{(1)} \\
& \vdots & \\
& \vdots & \\
\overline{\mathbf{A}}_{f_{L}, \theta_{1}}^{(M)} & \cdots & \overline{\mathbf{A}}_{f_{L}, \theta_{N}}^{(M)}
\end{array}\right) \mathbf{p}_{e}+\mathbf{n}_{e} \\
& =\mathbf{A}_{e} \mathbf{p}_{e}+\mathbf{n}_{e},
\end{aligned}
$$

where $\mathbf{n}_{e}$ is the vector obtained by deleting the elements in $\operatorname{vec}\left(\mathbf{R}_{n}\right)$ that correspond to the trivial zero elements in (12). Also, $\overline{\mathbf{A}}_{f_{l}, \theta_{n}}^{(m)}$ is the $m$ th column vector of the matrix $\overline{\mathbf{A}}_{f_{l}, \theta_{n}}$ defined as

$$
\overline{\mathbf{A}}_{f_{l}, \theta_{n}}=\mathbf{a}_{f_{l}, \theta_{n}} \mathbf{a}_{f_{l}, \theta_{n}}{ }^{H} .
$$

Here, $\mathbf{a}_{f_{i}, \theta_{n}}$ is the $M$-dimensional mode vector, and each element is defined by (2). From the above, it is seen that we can estimate the DOA of multi-band signals using the CS technique because $\mathbf{r}_{e}$ is the linear transform and $\mathbf{p}_{e}$ is the sparse vector representing the powers of impinging waves.

\subsection{Generally Frequency Dependent Case}

In real environments, received powers in the bands are different because transmitter outputs and/or propagation paths in general have frequency characteristics. Since we assumed that the powers of the signals in all the bands are the same, 
as given by (14), we cannot estimate DOAs correctly using the scheme proposed in the previous subsection. In this subsection, we extend the technique in such a way that it can estimate DOAs of signals having different complex amplitudes in the bands. The received power at the $m$ th antenna in the $f_{l}$ band can be expressed as

$$
\begin{aligned}
Q_{f_{l}, m} & =E\left[\left|y_{f_{l}, m}(s)\right|^{2}\right] \\
& =\left|x_{f_{l}, \theta_{1}}\right|^{2}+\cdots+\left|x_{f_{l}, \theta_{N}}\right|^{2}+\left|n_{f_{l}, m}\right|^{2} .
\end{aligned}
$$

We can observe the received signal $y_{f_{l}, m}(s)$ in each band using a narrow band filter, as shown in Fig. 3. If incident waves have frequency characteristics, the received powers vary from band to band. We can adjust the received signals in such a way that they have the same power in all the bands. The adjusted received signal vector in the $f_{l}$ band is given by

$$
\mathbf{y}_{f_{l}}^{\prime}(s)=\left(\frac{1}{\sqrt{Q_{f_{l}, 1}}} y_{f_{l}, 1}(s), \cdots, \frac{1}{\sqrt{Q_{f_{l}, M}}} y_{f_{l}, M}(s)\right)^{T} .
$$

This has the same power in all the bands if we ignore the noise power, and it is possible to use the technique presented in the previous subsection. It is seen that (19) can be expressed as

$$
\mathbf{r}_{e}^{\prime}=\mathbf{A}_{e} \mathbf{p}_{e}^{\prime}+\mathbf{n}_{e}^{\prime},
$$

where $\mathbf{p}_{e}^{\prime}$ is the vector of the adjusted incident powers, and $\mathbf{r}_{e}^{\prime}$ and $\mathbf{n}_{e}^{\prime}$ correspond to $\mathbf{r}_{e}$ and $\mathbf{n}_{e}$, respectively. Then, we can apply the CS technique to (23) in the same manner as to (19).

\section{Evaluation by Computer Simulation}

\subsection{Simulation Conditions}

We carried out computer simulations to evaluate the performance of the schemes proposed in the previous section. The parameters of the simulations are listed in Table 1. The center frequencies of the bands are $f_{1}=3.0 \mathrm{GHz}, f_{2}=4.5 \mathrm{GHz}$, $f_{3}=6.0 \mathrm{GHz}, f_{4}=7.5 \mathrm{GHz}$, and $f_{5}=9.0 \mathrm{GHz}$, and the antenna spacing is $0.05 \mathrm{~m}$, half the wavelength for $3.0 \mathrm{GHz}$. If the number of bands is increased, the performance can be improved because of the dimension extension. However, when the frequency separation between bands is very

Table 1 Simulation parameters

\begin{tabular}{c|c}
\hline \multicolumn{2}{c}{ Basic parameters } \\
\hline Antenna array & ULA \\
Number of antenna elements & 5 \\
Antenna separation [m] & 0.05 \\
DOA & Uniform distribution from $-90^{\circ}$ to $90^{\circ}$ \\
Number of divisions of angle & 181 \\
Frequency bands [GHz] & $3.0,4.5,6.0,7.5,9.0$ \\
Number of trials & 1000 \\
\hline \multicolumn{2}{c}{ Parameters for compressed sensing } \\
\hline$\mu$ & 10.0 \\
$\epsilon, p$ & $1.0 \times 10^{-4}$ \\
\hline
\end{tabular}

small, the performance improvement may be reduced because phase differences between antenna elements for signals in those bands are almost the same. Detailed analysis about this is our future work.

We determined the transmit signals $\mathbf{x}(s)$ using $M$ sequence random numbers. Different intervals in the $M$ sequence random numbers were used for signals arriving from different angles, and they were uncorrelated. The number of divisions of the angle, that is, the number of bins is 181 . We compared the performance of the proposed methods with that of the MUSIC algorithm and that of the CS technique for single-band signals. The center frequency for those methods was $3.0 \mathrm{GHz}$. Parameters $\mu, p$, and $\epsilon$ for the CS techniques were empirically determined as $\mu=10.0, p, \epsilon=1.0 \times 10^{-4}$.

We calculated probabilities of correct estimation of DOAs and root mean square errors (RMSEs). Correct estimation of DOA was defined as the case where the partition bins of the true directions and the estimated directions are completely the same. The RMSE in the simulations was defined as

$$
\operatorname{RMSE}=\frac{1}{K} \sum_{k=1}^{K} \sqrt{\frac{1}{T} \sum_{t=1}^{T}\left|\hat{\theta}_{k, t}^{\prime}-\theta_{k}{ }^{\prime}\right|},
$$

where $K$ is the number of arriving waves, $T$ denotes the number of trials, $\hat{\theta}_{k, t}^{\prime}$ is the estimated DOA of the $k$ th wave in the $t$ th trial, and $\theta_{k}{ }^{\prime}$ denotes the true DOA of the $k$ th wave.

\subsection{Results and Discussion}

First, we consider the case where the incident signals have the same power in all the bands. The phases alone are uniformly distributed.

DOA estimation results for the case of two incident waves as a function of SNR are shown in Fig. 5. The angles of incident waves are randomly distributed in the range from $-90^{\circ}$ to $90^{\circ}$. In the figure, the "Proposed CS" curve indicates the results of the proposed method using the CS technique with the Khatri-Rao operation applied to multiband signals. Since the two signals have the same power in all the bands, we did not adjust the powers. We estimated the DOAs using (19) in Sect. 3.1. "Multi-band CS" and "Single-band CS" represent the results of the conventional method for multi-band signals given by (11) and a single-band signal given by (1) without the Khatri-Rao operation, respectively. Also, "MUSIC" indicates the results of the conventional MUSIC method for a single-band signal. As stated previously, the conventional multi-band CS method cannot deal with signals whose phases are different in the bands. As for the method, we evaluated the performance under the condition that phases are the same in all the bands. The number of snapshots in each frequency band of "Proposed CS" and "Multi-band CS" is 20, and that of "Single-band CS" and "MUSIC" is 100 to make the total numbers of snapshots equal.

The proposed method had superior results to the con- 


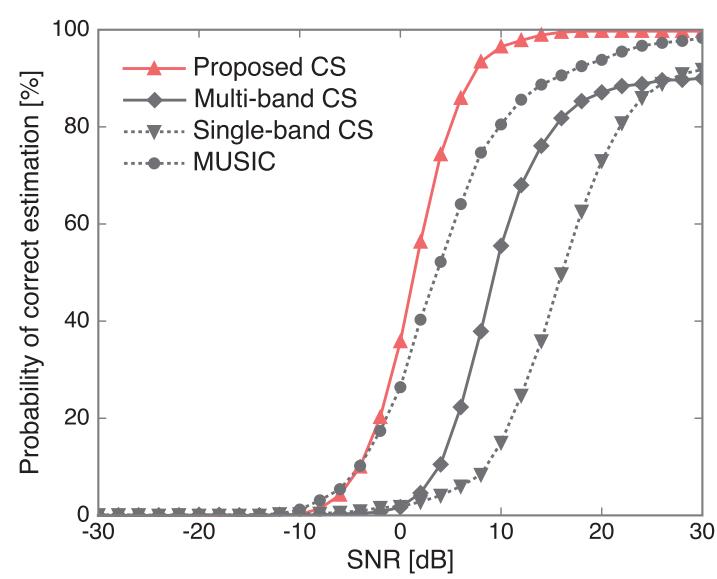

(a) Probability of correct estimation

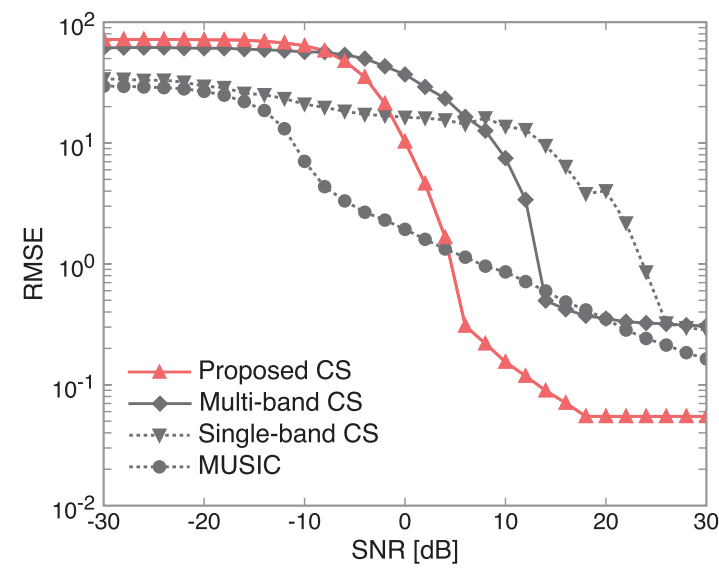

(b) RMSE

Fig. 5 DOA estimation results for two incident waves with equal power. SNR is the same in all the bands.

ventional methods. In particular, it achieved the lowest RMSE in the range from $6 \mathrm{~dB}$ to $30 \mathrm{~dB}$. The reason for its better performance than "Multi-band CS" is that the dimension of $\mathbf{r}_{e}$ for the proposed method using the Khatri-Rao operation $\left(M^{2} L\right)$ is greater than that of the received signal vector for "Multi-band CS" $(M L)$. The RMSE performance of the proposed method is, however, worse than "Single-band CS" and "MUSIC" in the lower SNR region. "Single-band CS" and "MUSIC" used 100 snapshots. On the other hand, the proposed method used 20 snapshots at each band. It is considered that the average of (13) using only 20 snapshots was not precise in the lower SNR region, and that the RMSE performance was worse in this region.

The probabilities of correct estimation as a function of the number of arriving waves with equal power are shown in Fig. 6. The number of waves ranged from one to ten, and the incident angles are randomly distributed from $-90^{\circ}$ to $90^{\circ}$. This is the same as in Fig. 5. The SNR is fixed at $20 \mathrm{~dB}$. In this case, the signals also have the same power in all the

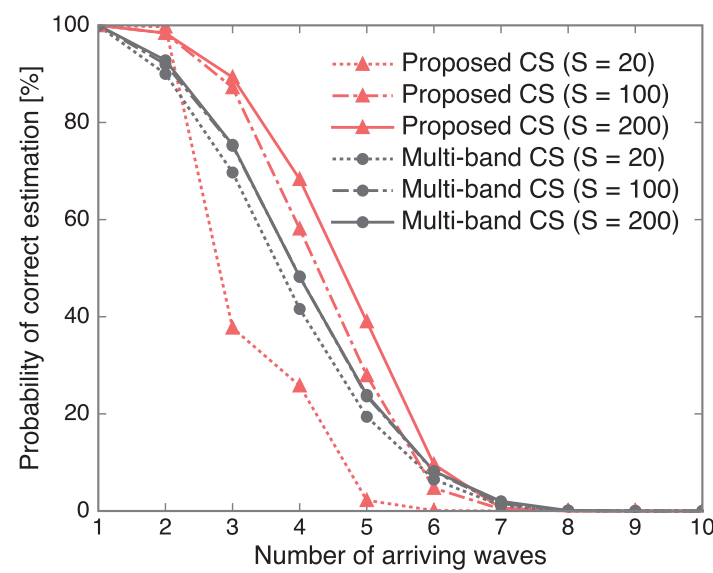

Fig. 6 Probabilities of correct estimation vs. number of arriving waves with equal powers. SNR is $20 \mathrm{~dB}$ in all the bands.

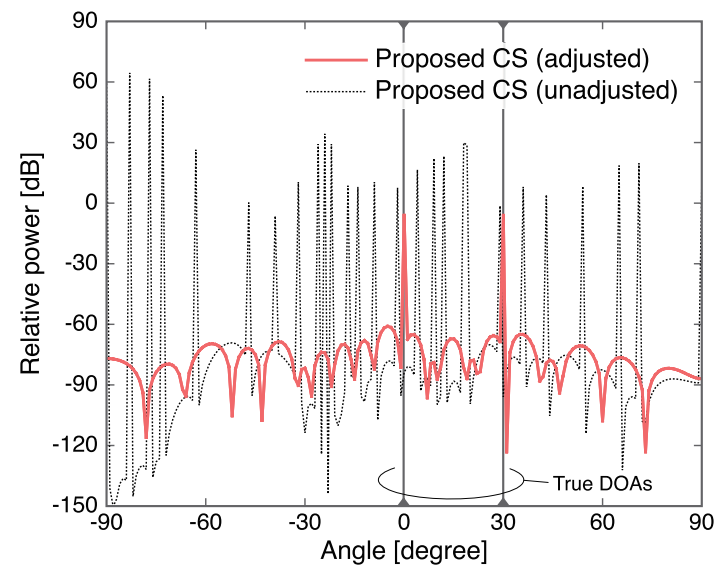

Fig. 7 Spectra obtained using the proposed methods for two incident signals with the same frequency characteristics $\left(\theta_{1}{ }^{\prime}=0^{\circ}, \theta_{2}{ }^{\prime}=30^{\circ}\right)$.

bands. The results for the proposed method and "Multi-band CS" are shown for cases where the numbers of snapshots $S$ in each band are 20,100, and 200. The results for "Multiband CS $(S=100)$ and "Multi-band CS $(S=200)$ are almost the same. It is seen that these methods can deal with cases where the number of waves exceeds the number of array elements. On the other hand, the probability of correct estimation by the proposed method is clearly smaller than that of "Multi-band CS" only when $S=20$.

The estimated relative power when two incident waves have frequency characteristics is shown in Fig. 7. The angles of the incident waves are $0^{\circ}$ and $30^{\circ}$, and we assumed that the frequency characteristics of both waves are the same. The SNRs in the bands $3.0 \mathrm{GHz}, 4.5 \mathrm{GHz}$, $6.0 \mathrm{GHz}, 7.5 \mathrm{GHz}$, and $9.0 \mathrm{GHz}$ are $35.4 \mathrm{~dB}, 31.9 \mathrm{~dB}$, $29.4 \mathrm{~dB}, 27.4 \mathrm{~dB}$, and $25.9 \mathrm{~dB}$, respectively. These frequency characteristics are determined by the rule of distance attenuation. We set the number of snapshots in each frequency band to 100. In this figure, "Proposed CS (adjusted)" and "Proposed CS (unadjusted)" indicate methods using (23) and (19), respectively. We can see that the re- 


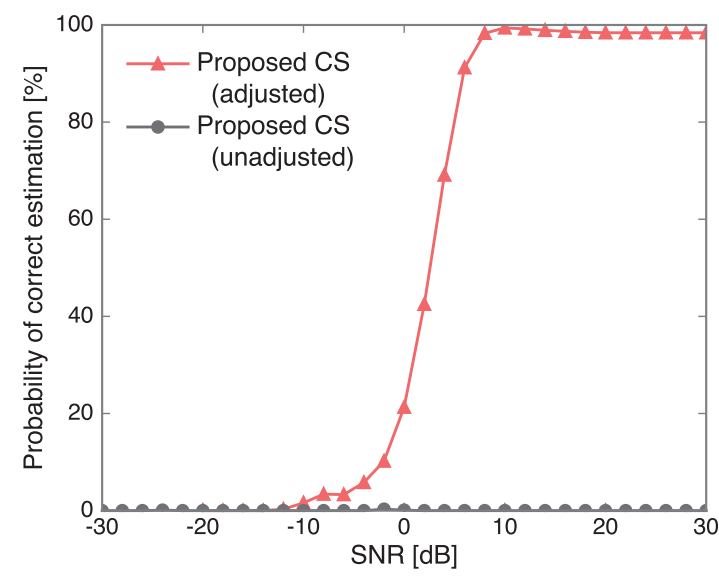

Fig. 8 Probabilities of correct estimation of DOAs for two signals with frequency characteristics.

sults of "Proposed CS (adjusted)" are perfectly correct. By contrast, "Proposed CS (unadjusted)" results have many incorrect peaks.

The probabilities of correct estimation of DOAs when two incident waves have the same power and same frequency characteristics are shown in Fig. 8. The abscissa indicates the average SNR over all the bands. The SNRs for the bands $3.0 \mathrm{GHz}, 4.5 \mathrm{GHz}, 6.0 \mathrm{GHz}, 7.5 \mathrm{GHz}$, and $9.0 \mathrm{GHz}$ are $5.4 \mathrm{~dB}, 1.9 \mathrm{~dB},-0.6 \mathrm{~dB},-2.6 \mathrm{~dB}$, and $-4.1 \mathrm{~dB}$ around the average SNR, respectively. The angles of the incident waves are uniformly distributed in the range from $-90^{\circ}$ to $90^{\circ}$. We can see that the adjusted proposed method shows significant superiority over the unadjusted one.

\section{Conclusion}

In this paper, we proposed methods of DOA estimation of frequency dependent multi-band signals using CS techniques. Evaluation by computer simulation showed that, although the proposed methods require more snapshots than conventional DOA estimation methods without the KhatriRao operation, the probability of correct estimation is much higher because of the extension of the dimension. In addition, the proposed method with power adjustment is not susceptible to frequency characteristics and it achieves higher estimation accuracy.

The relation between the performance of the proposed methods and the number of snapshots has not been fully clarified yet. This consideration is future work.

\section{Acknowledgement}

This work was supported by the Strategic Information and Communications R\&D Promotion Programme (SCOPE) under Grant 135001102 in 2013.

\section{References}

[1] T. Terada, T. Nishimura, Y. Ogawa, T. Ohgane, and H. Yamada, "DOA estimation of multi-band signals using a compressed sensing technique," Proc. IEEE iWAT 2014, pp.392-395, March 2014.

[2] L.C. Godara, "Application of antenna arrays to mobile communications, Part II: Beam-forming and direction-of-arrival considerations," Proc. IEEE, vol.85, no.8, pp.1195-1245, Aug. 1997.

[3] J. Capon, "High resolution frequency-wavenumber spectrum analysis," Proc. IEEE, vol.57, no.8, pp.1408-1418, Aug. 1969.

[4] R.O. Schumidt, "Multiple emitter location and signal parameter estimation," IEEE Trans. Antennas. Propag., vol.AP-34, no.3, pp.276280, March 1986.

[5] R. Roy and T. Kailath, "ESPRIT-estimation of signal parameters via rotational invariance techniques," IEEE Trans. Speech Signal Process., vol.37, no.7, pp.984-995, July 1989.

[6] M. Feder and E. Weinstein, "Parameter estimation of superimposed signals using the EM algorithm," IEEE Trans. Acoust. Speech Signal Process., vol.36, no.4, pp.477-489, April 1988.

[7] M.I. Miller and D.R. Fuhrmann, "Maximum-likelihood narrowband direction finding and the EM algorithm," IEEE Trans. Acoust. Speech Signal Process., vol.30, no.9, pp.1560-1577, Sept. 1990.

[8] J.A. Fessler and A.O. Hero, "Space-alternating generalized expectation-maximization algorithm," IEEE Trans. Signal Process. vol.42, no.10, pp.2664-2677, Oct. 1994.

[9] D.L. Donoho, "Compressed sensing," IEEE Trans. Inf. Theory, vol.52, no.4, pp.1289-1306, April 2006.

[10] E.J. Candes and M.B. Wakin, "An introduction to compressive sampling," IEEE Signal Process. Mag., vol.25, no.2, pp.21-30, March 2008.

[11] M. Cetin, D.M. Malioutov, and A.S. Willsky, "A variational technique for source localization based on a sparse signal reconstruction perspective," Proc. IEEE ICASSP, vol.3, pp.2965-2968, May 2002.

[12] M. Fujimoto and T. Hori, "Sub-band processing for DOA estimation of UWB," IEICE Commun. Express, vol.1, no.1, pp.23-27, June 2012.

[13] K. Hayashi, M. Nagahara, and T. Tanaka, "A user's guide to compressed sensing for communications systems," IEICE Trans. Commun., vol.E96-B, no.3, pp.685-712, March 2013.

[14] T. Terada, T. Nishimura, Y. Ogawa, and T. Ohgane, "DOA estimation of multi-band signals using a sparse signal reconstruction method," Proc. IEEE-APS/URSI International Symposium 2013, pp.866-867, July 2013.

[15] W.K. Ma, T.H. Hsieh, and C.Y. Chi, "DOA estimation of quasi- stationary signals with less sensors than sources and unknown spatial noise covariance: A Khatri-Rao subspace approach,” IEEE Trans. Signal Process., vol.58, no.4, pp.2168-2180, April 2010.

[16] S. Shirai, H. Yamada, and Y. Yamaguchi, "A novel DOA estimation error reduction preprocessing scheme of correlated waves for Khatri-Rao product extended-array," IEICE Trans. Commun. vol.E96-B, no.10, pp.2475-2482, Oct. 2013.

[17] P. Ciuciu, J. Idier, and J.F. Giovannelli, "Markovian high resolution spectral analysis," Proc. IEEE ICASSP, vol.3, pp.1601-1604, March 1999.

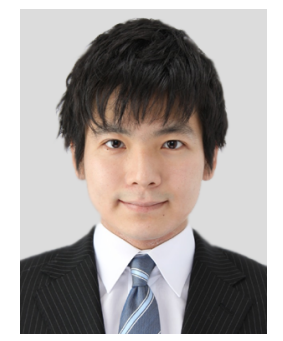

Tsubasa Terada received a B.E. degree in electronics and information engineering from Hokkaido University, Sapporo, Japan, in 2012. $\mathrm{He}$ is currently a master course student at the Graduate School of Information Science and Technology, Hokkaido University. His interests are in DOA estimation. 


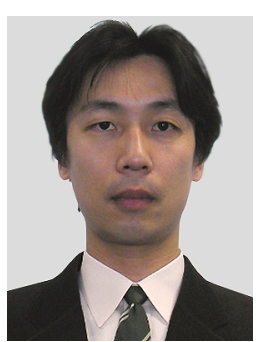

Toshihiko Nishimura received his B.S. in physics in 1992, his M.S. in physics in 1994, and his Ph.D. in electronics engineering in 1997 all from Hokkaido University, Sapporo, Japan. He joined the Graduate School of Engineering (now reorganized as the Graduate School of Information Science and Technology) at Hokkaido University in 1998, where he is currently an Assistant Professor of the Graduate School of Information Science and Technology. His current research interests are in MIMO systems using smart antenna techniques. He received the Young Researchers' Award of IEICE Japan in 2000, the Best Paper Award from IEICE Japan in 2007, and the Best Magazine Paper Award in 2011 from the IEICE Communications Society. He is a member of the IEEE.

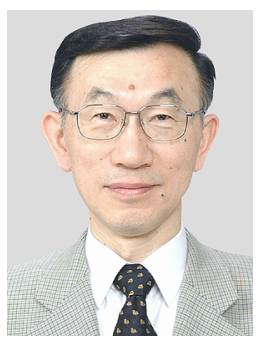

Yasutaka Ogawa received his B.E., M.E., and Ph.D. from Hokkaido University, Sapporo, Japan, in 1973, 1975, and 1978. Since 1979, he has been with Hokkaido University, where he is currently a full Professor at the Graduate School of Information Science and Technology. In 1992-1993, he was with the ElectroScience Laboratory of Ohio State University, U.S.A., as a Visiting Scholar, on leave from Hokkaido University. His interests are in adaptive antennas, mobile communications, super-resolution techniques, and MIMO systems. He received the Yasujiro Niwa outstanding paper award in 1978, the Young Researchers' Award of IEICE Japan in 1982, the Best Paper Award from IEICE Japan in 2007, and the Best Magazine Paper Award in 2011 from the IEICE Communications Society. He is a Fellow of the IEEE.

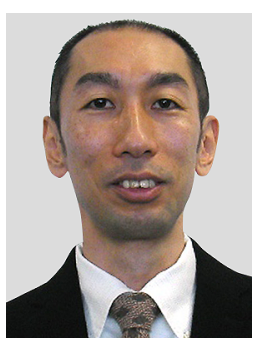

Takeo Ohgane received his B.E., M.E., and $\mathrm{Ph} . \mathrm{D}$. in electronics engineering from Hokkaido University, Sapporo, Japan, in 1984, 1986, and 1994. He was with the Communications Research Laboratory of the Ministry of Posts and Telecommunications from 1986 to 1992 . He was on assignment at the ATR Optical and Radio Communications Research Laboratory from 1992 to 1995 . Since 1995 , he has been with Hokkaido University, where he is an Associate Professor. He was at the Centre for Communications Research of the University of Bristol, U.K., as a Visiting Fellow in 2005-2006. His interests are in MIMO signal processing for wireless communications. He received the Young Researchers' Award of IEICE Japan in 1990, the IEEE AP-S Tokyo Chapter Young Engineer Award in 1993, the Best Paper Award from IEICE Japan in 2007, and the Best Magazine Paper Award in 2011 from the IEICE Communications Society. He is a member of the IEEE.

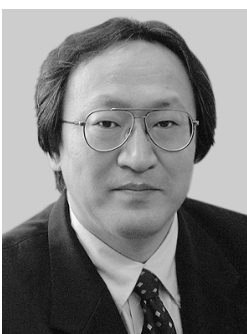

Hiroyoshi Yamada received the B.E., M.E. and Dr.Eng. degrees in electronic engineering from Hokkaido University, Sapporo, Japan, in 1988, 1990 and 1993, respectively. In 1993, he joined the Faculty of Engineering, Niigata University, where he is a professor. From 2000 to 2001, he was a Visiting Scientist at the Jet Propulsion Laboratory, California Institute of Technology, Pasadena, His current interests include superresolution techniques, array signal processing, and microwave remote sensing and imaging. He received the Young Engineer Award of IEEE AP-S Japan Chapter in 1992, the Young Engineer Award of IEICE Japan in 1999, the Kiyasu-Zenichi Award and the Best Paper Award of IEICE both in 2010, and the Best Tutorial Paper Award from Comm. Soc. of IEICE in 2010. He is a member of the IEEE. 